\section{Complementary \\ Medicine Research \\ Practice|Methods|Perspectives}

Society Bulletins - Gesellschaftsmitteilungen

Complement Med Res 2018;25:423-426

\section{SMGP

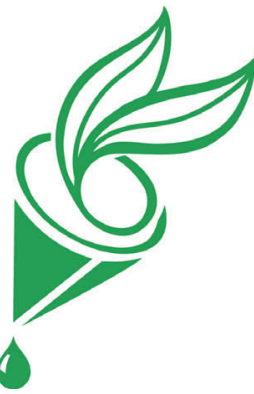

Korrespondenzadresse

Prof. Dr. sc. nat. Beat Meier

Geschäftsstelle SMGP

c/o Zürcher Hochschule für angewandte Wissenschaften

Fachgruppe Phytopharmazie und Naturstoffe

Grüental, Postfach 335, 8820 Wädenswil, Schweiz

Beat.Meier@zhaw.ch

\section{SMGP-Phytozirkel - ein Erfahrungsaustausch, der sich lohnt}

Vor einigen Jahren wurde von SMGP-Mitgliedern, insbesondere von jenen mit Zertifikat, immer wieder der Wunsch nach Arbeitsgruppen für einen phytotherapeutischen Erfahrungsaustausch geäussert.

Die Tagungen und Exkursionen sind zwar gute Gelegenheiten, um sich fachlich auszutauschen und fortzubilden sowie das phytotherapeutische Wissen zu vertiefen, aber das Bedürfnis nach einem niederschwelligen Angebot bedienen diese beiden Fortbildungsoptionen nicht.

Im Jahr 2003 bildete sich die Arbeitsgruppe Herbadonna, die jährlich mehrere PhytotherapieWorkshops (seit 2008 4-mal pro Jahr) zu gynäkologischen Fragestellungen durchführt ( $w w w$. herbadonna.ch).

Im September 2006 wurde dann der erste abendliche Phytozirkel in Zürich unter der Leitung von Peter Frey und Beatrix Falch (Porträts siehe CMR 5/2018 und CMR 6/2017) organisiert und durchgeführt. Das Ziel ist es, sich über phytotherapeutische Behandlungsmöglichkeiten von bestimmten - im Voraus ausgewählten - Indikationsgebieten auszutauschen und die verschiedenen Optionen zu diskutieren. Von jedem Phytozirkel wird ein Protokoll verfasst, in dem die phytotherapeutischen Vorschläge dokumentiert werden. Dieses Protokoll wird den Teilnehmenden des jeweiligen Phytozirkels zur Verfügung gestellt.

Bald folgten weitere 2-stündige, abendliche Phytozirkel: in Basel (zurzeit nicht aktiv), Bern (Leitung: Silvio Ballinari), St. Gallen (Leitung: Esther Granitzer), Lausanne (zurzeit nicht aktiv) und Luzern (Leitung: Christoph Bachmann). Zudem gibt es einen pädiatrischen Phytozirkel in Aarau (Leitung: Beatrix Falch). Sie alle sind ähnlich strukturiert und finden inzwischen je 2- bis 4-mal pro Jahr statt. Seit 2016 beginnen alle Phytozirkel mit einem Impulsreferat, dessen Thema und Inhalt dann anschliessend im Plenum diskutiert werden. Dieses neue Konzept hat sich bewährt, da auf diese Weise die Erfahrungen zu einem bestimmten Beschwerdebild sehr fundiert zusammengetragen und dokumentiert werden können.

Auch wenn die Phytozirkel ursprünglich für die bereits Zertifizierten vorgesehen waren, steht die Teilnahme allen an Phytotherapie interessierten Fachkreisen offen und kann auch im Rahmen des Fähigkeitsprogramms Phytotherapie der SMGP im Modul 5 (gilt für Ärztinnen/Ärzte) bzw. Modul 6 (gilt für Apothekerinnen/Apotheker) angerechnet werden.

Die Termine für die Phytozirkel finden sich auf der SMGP-Website in der Rubrik «Fähigkeitsprogramm» (https://www.smgp.ch/smgp/homeindex/ faehigkeitsprog.html). Weitere Informationen sind auch bei der SMGP-Vizepräsidentin, Beatrix Falch (b.falch@smgp.ch), erhältlich.

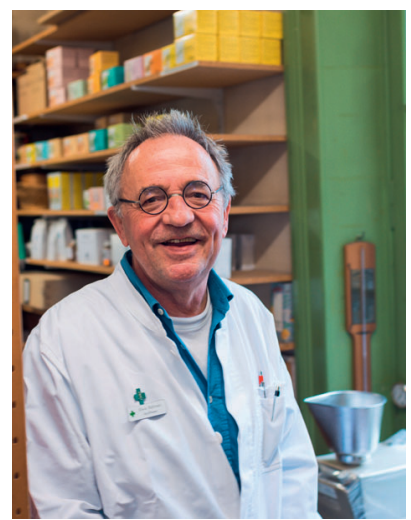

\title{
Silvio Ballinari,
}

Leitung SMGP-Phytozirkel Bern

1951 wurde ich als viertes Kind im Haus der Apotheke meiner Eltern geboren. Sie waren beide Apotheker, wie übrigens auch meine Schwester. Meine Mutter, die ursprünglich Botanikerin werden wollte, hat mein Interesse für Heilpflanzen geweckt. Am pharmazeutischen Institut in Bern habe ich von 19701976 studiert. 1981 habe ich die Apotheke meiner Eltern übernommen und mich immer mehr den natürlichen Heilmitteln zugewandt. In den Jahren 1986 bis circa 2000 durfte ich für die Pharmaziestudenten Vorlesungen über die fetten Öle und die aetherischen Öle sowie eine Einführung in die Homöopathie halten. Die Herstellung von phytotherapeutischen Magistralrezepturen verdanke ich den Ärztinnen, die diese verschrieben haben. Heute sind wir eine SMGP-Phytoreferenzapotheke. Die Phytotherapie eignet sich sehr für die individuelle Verschreibung von Magistralrezepturen und gewinnt zusehends an Bedeutung. Ich bin Mitglied der ALT-Kommission von pharmaSuisse. Da die herzustellenden Phytopräparate korrekt verschrieben werden sollten, ist der Kontakt mit den VerschreiberInnen wichtig. Die SMGP-Phytozirkel erlauben eine interdisziplinäre Zusammenarbeit und einen Erfahrungsaustausch mit den ÄrztInnen, die einzigartig sind. Wenn wir uns fortbilden wollen, müssen wir uns begegnen. Deshalb bin ich glücklich, diesen Berner Phytozirkel der SMGP zu leiten.

\section{KARGER}



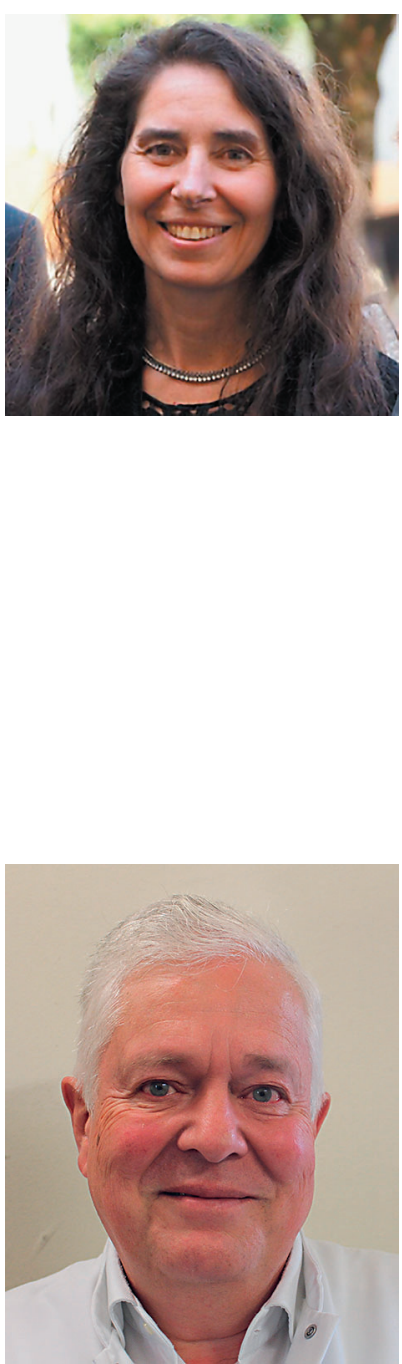

Dr. scient. med. Esther Granitzer,

Leitung SMGP-Phytozirkel St. Gallen

Als Komplementärmedizinerin MSc und approbierte Naturheilpraktikerin TEN führe ich schon seit fast 22 Jahren meine Praxis für Komplementärmedizin mitten in St. Gallen.

Mit Phytotherapie kam ich schon als Kind in Berührung, als ich meiner Grossmutter bei der Herstellung von Ringelblumensalbe und Arnikaschnaps half. Zu meinem 8. Geburtstag bekam ich dann von einer Nachbarin mein erstes Pflanzenbuch geschenkt, das mein Phytointeresse vollends weckte.

Nach verschiedenen Ausbildungen und Anstellungen im Schulmedizin-Bereich schloss ich das Studium zum «Master of Science in Complementary Medicine» an der Inter-Universität in Graz (Österreich) nach 3 Jahren mit der Masterarbeit zum Thema «Weizenwachstum unter dem Einfluss von phytotherapeutisch zubereitetem Hypericum perforatum» ab.

Das Studium der Medizinischen Wissenschaften an der Universität Fürstentum Liechtenstein UFL zum Dr. scient. med. dauerte ebenfalls 6 Semester. Ein Teil meiner Doktorarbeit zum Thema «Auswirkungen von Psyllium (Flohsamen) auf Parameter des Glukosestoffwechsels» wurde im 2017 in diesem Journal publiziert (Complement Med Res 2017;24:164-171; https://doi.org/10.1159/000477366).

Seit 2012 bin ich Mitglied bei der SMGP, und seit März 2014 findet der Phytozirkel St. Gallen unter meiner Leitung statt. Der regelmässige Austausch mit den Phytozirkelteilnehmern in meiner Praxis ist jedes Mal eine wertvolle Erfahrung, und ich freue mich immer sehr auf diesen gewinnbringenden Abend.

\section{Dr. pharm. Christoph Bachmann, \\ Leitung SMGP-Phytozirkel Luzern}

Zum Studium der Pharmazie kam ich eher zufällig über einen Umweg von 4 Semestern Germanistik. Ich bin kein typischer Naturwissenschaftler und versuche, wissenschaftliche Erkenntnisse über das Leben, Gesundheit und Krankheit mit einer ganzheitlichen Betrachtungsweise zu erklären.

Nach dem Pharmaziestudium (1981), einer pharmakologiehistorischen Dissertation (1985) und einigen Jahren Tätigkeit in meiner eigenen Apotheke kam ich zuerst mit der klassischen Homöopathie in Kontakt, und ich absolvierte einen entsprechenden Ausbildungsgang. Die traditionelle Pharmakognosie sprach mich weniger an. Die Therapie mit Vielstoffgemischen sagte mir aber schnell zu, und heute sehe ich mich als Pharmazeut, der versucht, zur Brückenbildung zwischen volksmedizinischen Erkenntnissen und moderner medizinischer Forschung beizutragen. Denn aus meiner ganzheitlichen Betrachtungsweise ist völlig klar, dass unsere Vorfahren aus Erfahrung lernten, Pflanzen zur Behandlung von Krankheiten zu nutzen.

Ich engagierte mich in der SMGP und war von 1997 bis 2016 Vorstandsmitglied. Daneben lebte ich meine geisteswissenschaftliche Ader aus und betätigte mich als Freelancer für Phytotherapie bei verschiedenen medizinischen Fachzeitschriften.

2016 gründete ich zusammen mit einigen Apothekerinnen, einem Arzt und mehreren Ärztinnen den SMGP-Phytozirkel Luzern, den ich seither leite. 


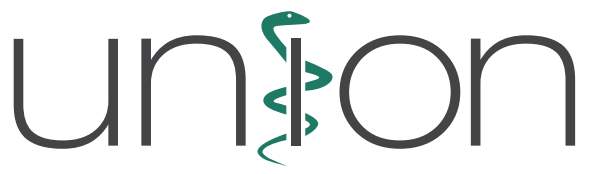

Schweizerischer komplementärmedizinischer Ärzteorganisationen des sociétés suisses de médecine complémentaire delle associazioni mediche svizzere di medicina complementare of associations of Swiss physicians for complementary medicine

Korrespondenzadresse / Correspondance à adresser à: Geschäftsstelle

UNION Schweizerischer komplementärmedizinischer

Arzteorganisationen

Tribschenstrasse 7, Postfach 3065

6002 Luzern

Tel.: +41 413685805

E-Mail: info@unioncomed.ch

Internet: https://www.unioncomed.ch

\section{UNION Schweizerischer komplementärmedizinischer Ärzteorganisationen}

\section{Zusammen stärker - die UNION Schweizerischer komplementär- medizinischer Ärzteorganisationen braucht es auch in Zukunft!}

\section{Mit der erfolgreichen Aufnahme der Komplementärmedizin in die medizini- sche Grundversorgung rückte die Schweiz als gutes Beispiel europaweit in den Fokus - Zusammenhalt unter den Disziplinen weiterhin sehr wichtig!}

Die Arbeit der UNION Schweizerischer komplementärmedizinischer Ärzteorganisationen ist auch in Zukunft sehr wichtig!

Mit der erfolgreichen Aufnahme der Komplementärmedizin in die medizinische Grundversorgung rückte die Schweiz als gutes Beispiel europaweit in den Fokus aber zunehmend gab es auch Kritik. Aktuell kommt es immer wieder zu medialen Angriffen - vor allem gegen die Homöopathie - in der Schweiz, aber auch in Europa.

Der kollegiale Zusammenhalt der 4 in der Grundversicherung der Schweiz verankerten komplementärmedizinischen Methoden «Anthroposophisch erweiterte Medizin», «Homöopathie», «Traditionelle Chinesische Medizin» und «Phytotherapie» ist deshalb auch in Zukunft sehr wichtig.

Komplementärmedizin ist wirksam! Das konnte in Studien mehrfach belegt werden, das erfahren wir komplementärmedizinisch tätigen Ärztinnen und Ärzte in unserer Praxistätigkeit jeden Tag, das erleben unsere Patienten, und deshalb ist die Komplementärmedizin in der Bevölkerung so beliebt und breit gestützt!

Ärztinnen und Ärzte mit einer Zusatzweiterbildung in Komplementärmedizin bekennen sich zur Wissenschaftlichkeit, und die Qualität der Forschungsarbeiten ist in den letzten Jahren wesentlich verbessert worden. Eine repräsentative Literaturstudie am Institut für Sozial- und Präventivmedizin der Universität Bern zu 89 placebokontrollierten Untersuchungen ergab z.B. für die Phytotherapie eine sogar tendenziell bessere Qualität der Studien als für die konventionelle Medizin [1].

Der aktuell viel zitierte australische Bericht, der sich gegen die Homöopathie richtet, ist wissenschaftlich umstritten. Zum Beispiel wurde die Datenerhebung 2-mal durchgeführt, und der erste Bericht wurde weder publiziert noch der Öffentlichkeit zur Verfügung gestellt.

Patienten und Ärzte sind zufrieden mit der Wirksamkeit und der Verträglichkeit komplementärmedizinischer Arzneimittel. Deshalb werden sie auch so gerne bei Säuglingen, Kindern, Schwangeren, Menschen höheren Alters und Menschen mit Mehrfachdiagnosen angewandt.

Der diesjährige Vergleich der SASISZahlen zeigt, dass Ärztinnen und Ärzte mit einem Fähigkeitsausweis in 1 der 4 Fachrichtungen der Komplementärmedizin im Vergleich kostenneutral arbeiten.

Die UNION setzt sich aktiv in der Strategie Antibiotikaresistenzen (StAR) des Bundes ein.

Die ärztliche Komplementärmedizin hat in der Behandlung von Infektionskrankheiten traditionsgemäss langjährige Erfahrungen im sparsamen, aber trotzdem sachgerechten Einsatz von Antibiotika. Nach neusten Zahlen der SASIS (von Dakomed in Auftrag gegeben) verwenden Grundversorger in der Schweiz mit einer Weiterbildung in Komplementärmedizin die Hälfte an Antibiotika im Vergleich zu Grundversorgern ohne eine solche Weiterbildung.

Eine Verzögerung der Antibiotikaverschreibung bei Infekten der Luftwege, wie sie in verschiedenen Leitlinien empfohlen wird, in Kombination mit effektiven und sicheren, nichtantibiotischen, z.B. komplementärmedizinischen Behandlungsmöglichkeiten ist eine sinnvolle Strategie zur
Senkung der Antibiotika-Verschreibungspraxis. Deshalb setzt sich die UNION dafür ein, dass Komplementärmedizin zumindest in die Supplemente zu den Leitlinien der Schweiz Eingang findet - wie es bei den Nutztieren bereits in Arbeit ist.

Komplementärmedizin ist in der $\mathrm{Be}$ völkerung beliebt, und die Nachfrage nach Ärztinnen und Ärzten mit einer Weiterbildung in Komplementärmedizin übersteigt das Angebot! Deshalb engagiert sich die UNION weiterhin gemeinsam in der Förderung des ärztlichen Nachwuchses. Auch dieses Jahr hatte die UNION einen Stand auf dem MEDIfuture-Kongress in Bern und konnte dort mit jungen Kolleginnen und Kollegen in Kontakt treten und sie für unseren Beruf begeistern.

Mit grosszügiger Unterstützung des Karger Verlags bleibt die Zeitschrift COMPlementary Medicine RESEARCH auch 2019 das Mitteilungsorgan der UNION. Die Mitglieder erhalten einen Rabatt von 50\% für den Bezug eines Individualabonnements. Die jeweiligen Mitteilungen werden aber auch weiterhin auf der Homepage der UNION veröffentlicht. Eine Auffrischung der Homepage ist in Arbeit.

\section{Dr. med. Gisela Etter}

Präsidentin UNION Schweizerischer komplementärmedizinischer

Ärzteorganisationen

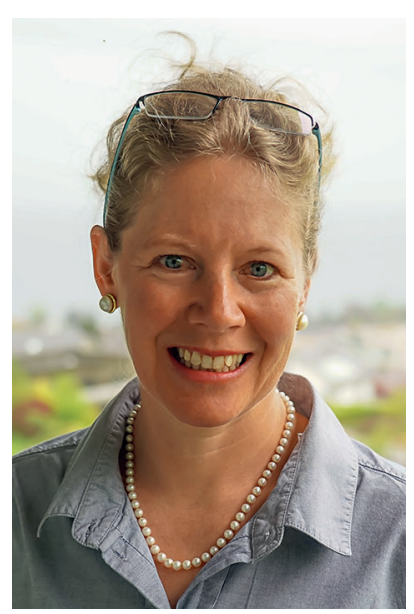

\section{Literatur}

1 Nartey L, Huwiler-Müntener $\mathrm{K}$, Shang $\mathrm{A}$, et al: Matched-pair study showed higher quality of placebocontrolled trials in Western phytotherapy than conventional medicine. J Clin Epidemiol 2007;6:787-794. 


\section{UNION des associations médicales suisses en médecine complémentaire}

\section{Plus forts ensemble - A l'avenir, I'UNION des sociétés suisses de médecine complémentaire doit rester soudée!}

\section{L'intégration réussie de la médecine complémentaire dans la médecine de premier recours a fait de la Suisse un bon exemple européen: la cohésion entre les disciplines reste essentielle!}

Le travail de l'UNION des sociétés suisses de médecine complémentaire est aussi très important dans l'avenir!

L'intégration réussie de la médecine complémentaire dans la médecine de premier recours a fait de la Suisse un bon exemple européen, mais a également intensifié la critique de ses détracteurs. En Suisse et en Europe, nous ne cessons de faire face à des attaques dans les médias, notamment contre l'homéopathie.

C'est pourquoi, à l'avenir, la cohésion des quatre méthodes de médecine complémentaire ancrées dans l'assurance de base en Suisse, à savoir la médecine élargie par l'anthroposophie, l'homéopathie, la médecine traditionnelle chinoise et la phytothérapie, reste essentielle.

La médecine complémentaire est efficace! De nombreuses études l'ont prouvé. Nous médecins, exerçant la médecine complémentaire au quotidien dans notre cabinet, nous en faisons l'expérience tous les jours et nos patients aussi. C'est pourquoi la médecine complémentaire est autant appréciée et soutenue par la population.

Les médecins ayant suivi une formation postgraduée supplémentaire en médecine complémentaire revendiquent le caractère scientifique de leur pratique. Ces dernières années, la qualité des travaux de recherche s'est d'ailleurs nettement améliorée. Une étude bibliographique représentative, réalisée par l'Institut de médecine sociale et préventive de l'université de Berne sur 89 études en double aveugle avec placebo a par exemple montré que les études sur la phytothérapie avaient tendance à être de meilleure qualité que celles portant sur la médecine conventionnelle [1].
Le rapport australien contre l'homéopathie fréquemment cité actuellement fait l'objet d'une controverse scientifique. Les données ont été deux fois collectées et le premier rapport n'a été ni publié, ni mis à la disposition de l'opinion publique.

Patients et médecins sont satisfaits de l'efficacité des médicaments de la médecine complémentaire, qui sont bien tolérés. C'est la raison pour laquelle ces traitements sont volontiers utilisés sur les nourrissons, enfants, femmes enceintes, personnes très âgées et patients atteints de multimorbidité.

La comparaison des chiffres fournis cette année par la société SASIS SA montre que les médecins titulaires d'une attestation de formation complémentaire dans l'une des quatre disciplines de la médecine complémentaire coûtent la même chose.

L'UNION s'implique activement dans la Stratégie Antibiorésistance Suisse (StAR) de la Confédération.

Traditionnellement, dans le traitement des maladies infectieuses, la médecine complémentaire applique depuis de longues années une politique d'utilisation parcimonieuse mais appropriée des antibiotiques. D'après les derniers chiffres de SASIS SA (demandés par Dakomed), en Suisse, les médecins de premier recours ayant suivi une formation postgraduée en médecine complémentaire utilisent moitié moins d'antibiotiques que ceux non formés dans ce domaine.

Une stratégie judicieuse pour faire baisser la prescription classique d'antibiotiques: retarder la prescription des antibiotiques en cas d'infection des voies respiratoires, comme c'est recommandé dans certaines directives, en associant d'éventuels traitements efficaces et sûrs, qui ne sont pas à base d'antibiotiques, comme les thérapies de médecine complémentaire. C'est pourquoi L'UNION s'engage pour que la médecine complémentaire soit au moins mentionnée dans les suppléments des directives suisses, comme dans la démarche en cours pour les animaux de rente.

La médecine complémentaire est appréciée par la population et la demande de médecins ayant suivi une formation postgraduée dans ce domaine dépasse l'offre! C'est pourquoi l'UNION continue de défendre avec cohésion la promotion de la relève médicale. Cette année encore, l'UNION a animé un stand à la foire
MEDIfuture à Berne, où elle a pu entrer en contact avec de jeunes collègues et les enthousiasmer sur notre profession.

Grâce au soutien généreux des éditions Karger, la revue Complementary Medicine Research restera en 2019 l'organe d'information de l'UNION. Les membres bénéficient d'une réduction de $50 \%$ sur un abonnement individuel. Mais toutes les communications continuent d'être publiées sur la page d'accueil de l'UNION. Nous sommes en train de travailler à la modernisation de la page d'accueil.

\section{Dr. méd. Gisela Etter}

Présidente de l'UNION des sociétés suisses de médecine complémentaire

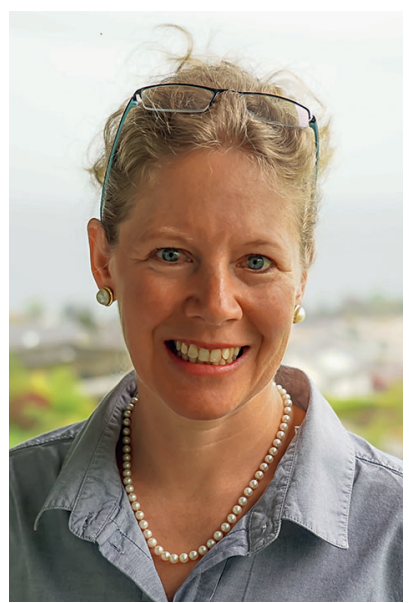

\section{Littérature}

1 Nartey L, Huwiler-Müntener K, Shang A, et al Matched-pair study showed higher quality of placebocontrolled trials in Western phytotherapy than conventional medicine. J Clin Epidemiol 2007;6:787-794. 